\section{Measuring Electron Intensities from Film}

Michael Lamvik

Measuring electron intensity is sometimes a useful thing to do as a part of an electron microscope study. The electron intensity recorded through a sample is one of the important pieces of information needed in determining the thickness of a thin film or the molecular weight of biological structures by electron scattering methods. Modern digital imaging systems in transmission electron microscopes allow relatively easy determination of electron intensities, but some labs don't have the expensive digital systems. Photographic film has been used traditionally to record electron microscope pictures, and film can record a very high density of spatial information. It is just a little more work to derive the electron intensity from an image on film than from a digital detector. For those labs interested in using film for preliminary measurements, or for student projects, I'd like to review some methods that have been known for years for making quantitative measurements from film.

The density $\mathrm{D}$ of an area of film that has received an exposure "I" to electrons in a TEM is given by

$$
D=D_{\max } \quad(1-\exp (-\mathrm{al}))
$$

where $a$ is a constant. When $D$ is much less than $D_{\max }$, the exponential can be expanded and truncated to the first term, yielding

\section{$\mathrm{D}=\mathrm{kE}$}

where $\mathrm{k}$ is a constant (Zeitler, 1992, Ultramicroscopy 46, 405).

Experimental data showing the linearity of the film re-

\section{Modern Coating Solutions for Today's EM Sample Prep Needs}

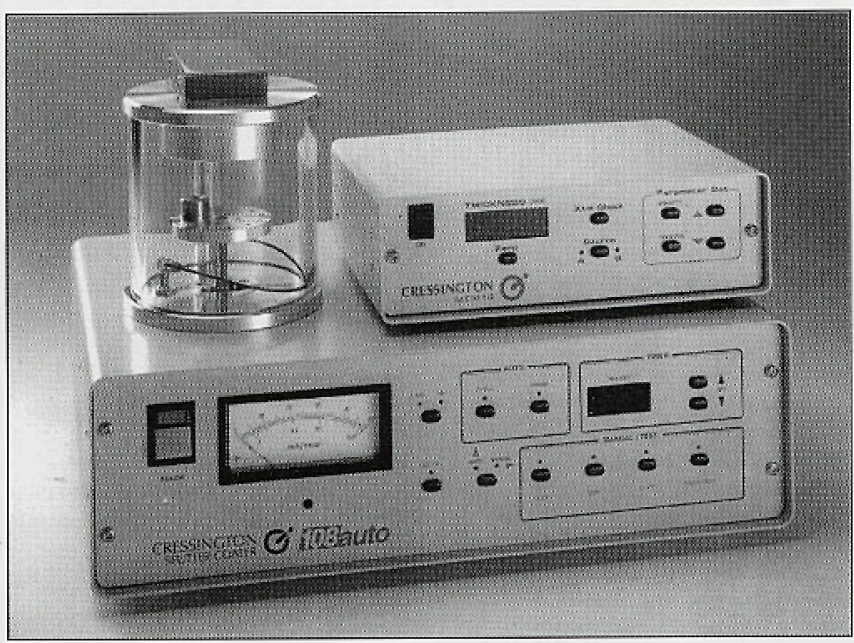

Compact modern desktop systems with fast cycle times. Carbon and sputtering systems for all SEM, FE-SEM and TEM applications.

\section{CRESSINGTON}

Cressington Scientific Instruments, Inc.

508 Thomson Park Drive, Cranberry Twp., PA 16066

TEL 800-755-1781 / 724-772-0220 FAX 724-772-0219

Website: www.cressington.com sponse for electrons is given in figure 4 of the paper cited. This paper notes that the linearity is valid for $D_{\max }$ less than 1.5 for all commercially available TEM emulsions. It is assumed here that the base and fog density of the film "d" has been subtracted out. For practical work, it is useful to be explicit about the base and fog density, yielding the equation

$$
D=k E+d \text {. }
$$

To use this equation in a practical way, the specimen to be measured is placed on a holey film. When the image is made, $D_{\max }$ is measured from the image of the hole on the film (because this is where the total beam current is recorded). $D$ is measured from any desired point on the image of the specimen. The base and fog density $d$ is measured from the shadow created by the rim of the film holder, or anyplace else that the film isn't exposed to electrons (assuming you don't have a high-voltage microscope, where the electrons might even penetrate the film holder).

Because all of the measurements are made on the same piece of film, the degree of development or the age of the film are not relevant because there doesn't need to be any comparison to any other piece of film.

The exposure time in the electron microscope should be set so that the film has a density range from something slightly above zero to something slightly above one. This range assures the linearity of the density with respect to the incident electron exposure. This is also a range that is easily measured with an analog densitometer. Because all of the measurements are made on the same piece of film with the same densitometer, the type of illumination or degree of collimation of the light in the densitometer is not relevant.

It's often not easy to find a densitometer in a contemporary lab, but it is getting more common to find transparency scanners, which are typically used to digitize films and slides for presentations. I used a Kodak \#2 Photographic Step Tablet to check the transparency scanner in the building where I work (this step tablet has 21 uniformly-spaced transmission density steps, which makes it easy to check linearity).

I found that our scanner was not bad, but the deviation from linearity of the scanner was worse than the deviation of the film response (given by the approximation $D=k E+d$, given above). This scanner has a density range limited to 8 bits, and the maximum density is also limited. Some labs have scanners with 10,12 or 16 bits of dynamic range for gray scale, and the wider range is useful. In any case, it is important to check the linearity of the scanner with something like the step tablet.

If the scanner is not linear, it is possible to measure the deviation with a calibrated density standard and correct the output images. In practical terms, the density standard (like the step tablet) should be scanned at the same time, in the same field-of-view, as the film being studied, to avoid creating any variables of illumination or scaling between scans.

As noted above, these methods aren't as easy as getting a picture from a digital imaging system. But sometimes it's very useful to be able to think quantitatively about images without having to wait for a big investment in a digital system.

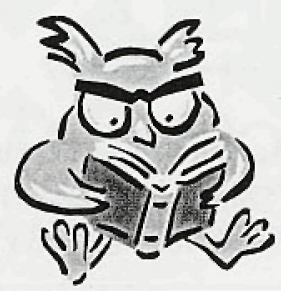




\section{Improve Your VPSEM Secondary Imaging Performance With....}

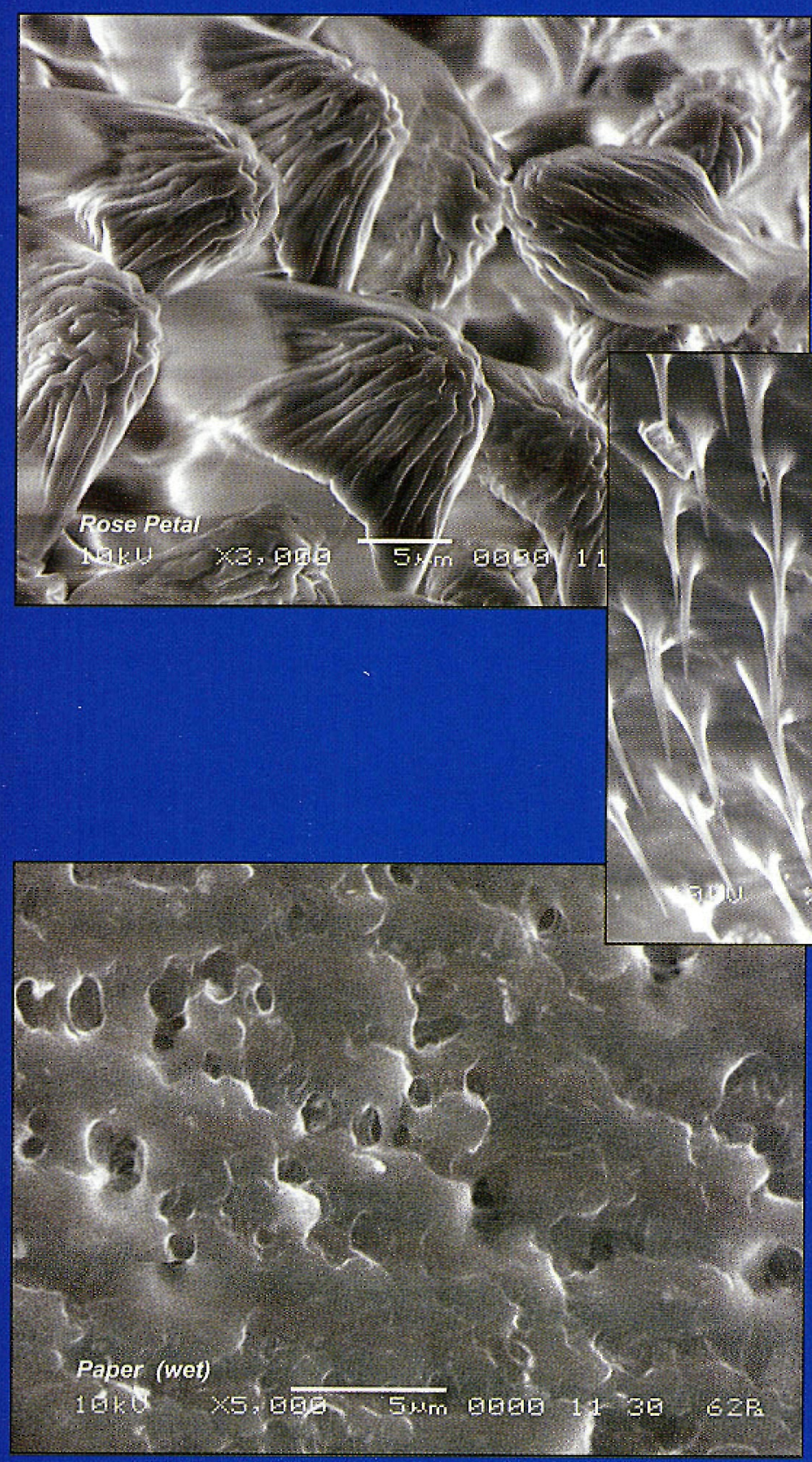

At last-Secondary Electron Imaging for your VPSEM! AMT's new MAXSEM

Detector will enhance the performance of your new or existing instrument.

High Sensitivity

Rapid Scan Rates

Retrofittable To All SEMs

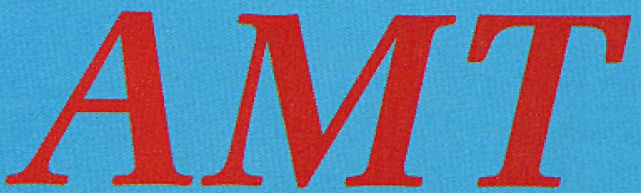

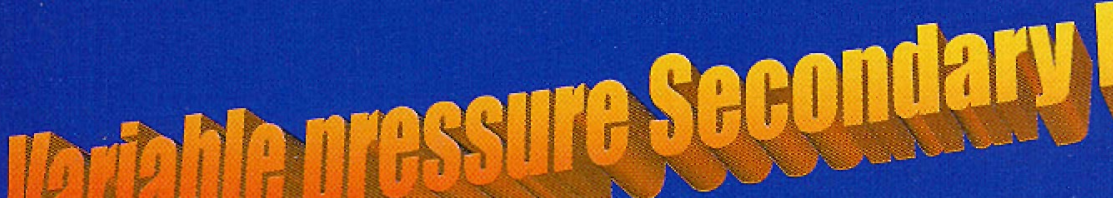

Advanced Microscopy Techniques 3 Electronics Avenue Danvers, MA 01923

Tel: (978)774-5550 Fax: (978)739-4313

Email:amtcorp@delphi.com

http://www.msa.microscopy.com/ amt/ 